\title{
Oromandibular Dystonia - A Purely Clinical Diagnostic Case Report
}

\author{
Saraswathi Gopal K* and Vani Anusha A \\ Department of oral medicine and radiology, Meenakshi ammal dental college, India
}

*Corresponding author: Saraswathi Gopal K, Professor and head of the department, Department of oral medicine and radiology, Meenakshi ammal dental college, Chennai -95, India, Email: dr.saraswathik@yahoo.co.in

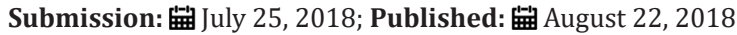

\begin{abstract}
Dystonia is the manifestation of involuntary lasting severe muscle contractions, which lead to rhythmic and atypical movements in different parts of the body. Dystonia is the most common movement disorder next to Parkinson's disease (PD) and essential tremor (ET). Oro Mandibular Dystonia (OMD) is considered as a focal dystonia involving mouth, jaw, and tongue, manifested by involuntary muscle contractions producing repetitive, patterned movements of the involved structures. The diagnosis of OMD is purely clinical and is to be differentiated thoroughly from the conditions mimicking the signs. Since it presents in various forms and severities it further renders the management a multidisciplinary approach with variable treatment outcomes. The following is a clinical diagnostic case report of oromandibualar dystonia with presenting signs and symptoms, history and examination characteristic of the condition.
\end{abstract}

Keywords: Dystonia; Cranifacial dystonia; Invoulantary; Muscle contractions; Multi-disciplinary management

Abbreviations: OMD: Oromandibular Dystonia; BoNT: Botulinum Neurotoxin; EMG: Electromyography; TMJ: Temporomandibular Joint; CNS: Central nervous system

\section{Introduction}

Oromandibular dystonia is thought to originate from a dysregulation of centrally mediated movement, whereby repetitive or sustained spasms of the masticatory, facial, or lingual muscles result in involuntary, and possibly painful jaw movement. Cranial dystonia is a broad description for dystonia that affects any part of the head [1]. Dystonia that affects the facial muscles and lips of musicians who play wind instruments is called embouchure dystonia [2,3]. Dystonia that specifically affects the tongue is called lingual dystonia. Oromandibular dystonia may be primary or secondary based on etiology [2]. Although the condition is rare with prevalence of 3.4 per million, it is often misdiagnosed or left undiagnosed due to several patient-clinician factors. The following is a diagnostic case report of focal dystonia of a dental patient who presented with apparently no subjective symptoms.

\section{Case Report}

A 58 year old female patient reported to the department of oral medicine and radiology at Meenakshi Ammal Dental College, Chennai with a chief complaint of pain in the left lower back tooth for the past one month. Patient gave a history of continuous and throbbing pain that was relieved on medication for two days which was prescribed by a dental surgeon in patients locality. Patient is a known hypertensive for the past one year and is under regular medication for the same. Patient gave a history of intermittent pain in the back of neck associated with occasional dizziness for which patient uses mechanical aids during sleep (cervical collar) for the past one year. Patient has undergone extraction of carious tooth one month before the time of presentation and has noticed a change in the pattern of her bite following extraction which did not provoke any painful symptoms or discomfort. No history of parafunctional habits or trauma in the craniofacial region were reported by the patient. Patient had a habit of chewing betel quid (with betel leaf, betel nut and slaked lime) for 40 years and has quit the habit one year back. On clinical examination, no gross asymmetry or structural deformities were elicited (Figure 1). Regular, rhythmic, repetitive, involuntary movements of the mandible mimicking habitual grinding of the teeth were evident. No apparent abnormality was detected on thorough examination of temporomandibular joints on right and left sides along with the examination of muscles of mastication. On intra oral examination, poor oral hygiene with extensive extrinsic stains were elicited. Edentulous region in relation to 36 was present. Generalized attrition of the teeth was characteristically present and sharp lingual cusps of 46 was present (Figure $2 \& 3$ ). Tenderness on percussion was elicited in 35 which was the offending tooth that the patient complained about. An orthopantamogram to evaluate 
the TMJ and the dentofacial complex was adviced. OPG did not reveal significant radiographical pathologies. Dentition appeared with occlusal reduction in the coronal height involving enamel and dentin suggestive of generalized attrition. Generalized crestal bone loss was evident suggestive of generalized periodontitis and apical widening of periodontal ligament space with loss of lamina dura in 35 suggestive of chronic apical periodontitis in 35 (Figure 4). Normal joint spaces with apparently normal condylar anatomy was seen. From the clinical findings of involuntary and repetitive movements, a diagnosis of oromandibular dystonia was considered.

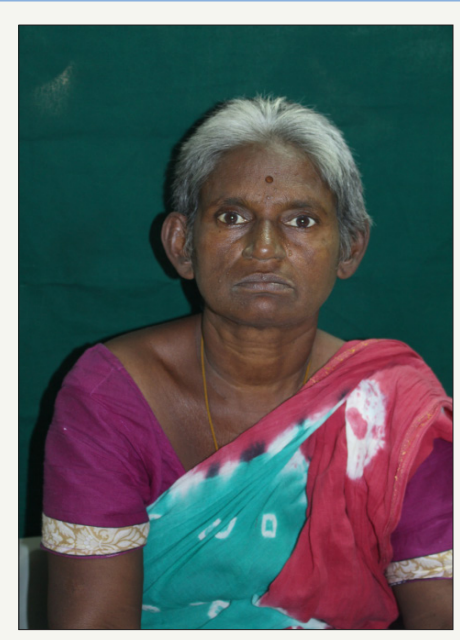

Figure 1: Patient profile.

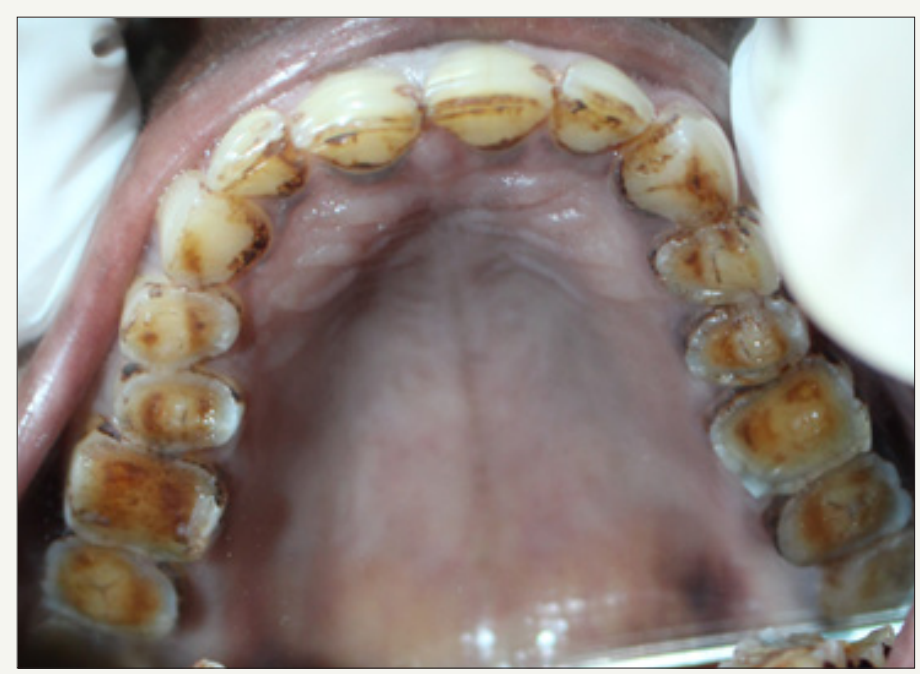

Figure 2: Maxillary dentition showing severe attrition and extrinsic stains.

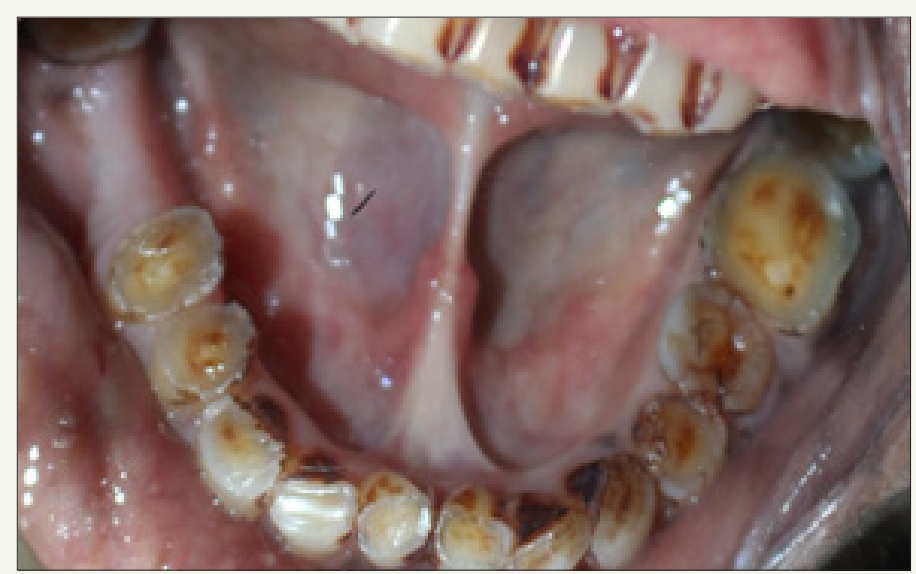

Figure 3: Mandibular dentition with severe attrition and extrinsic stains. 


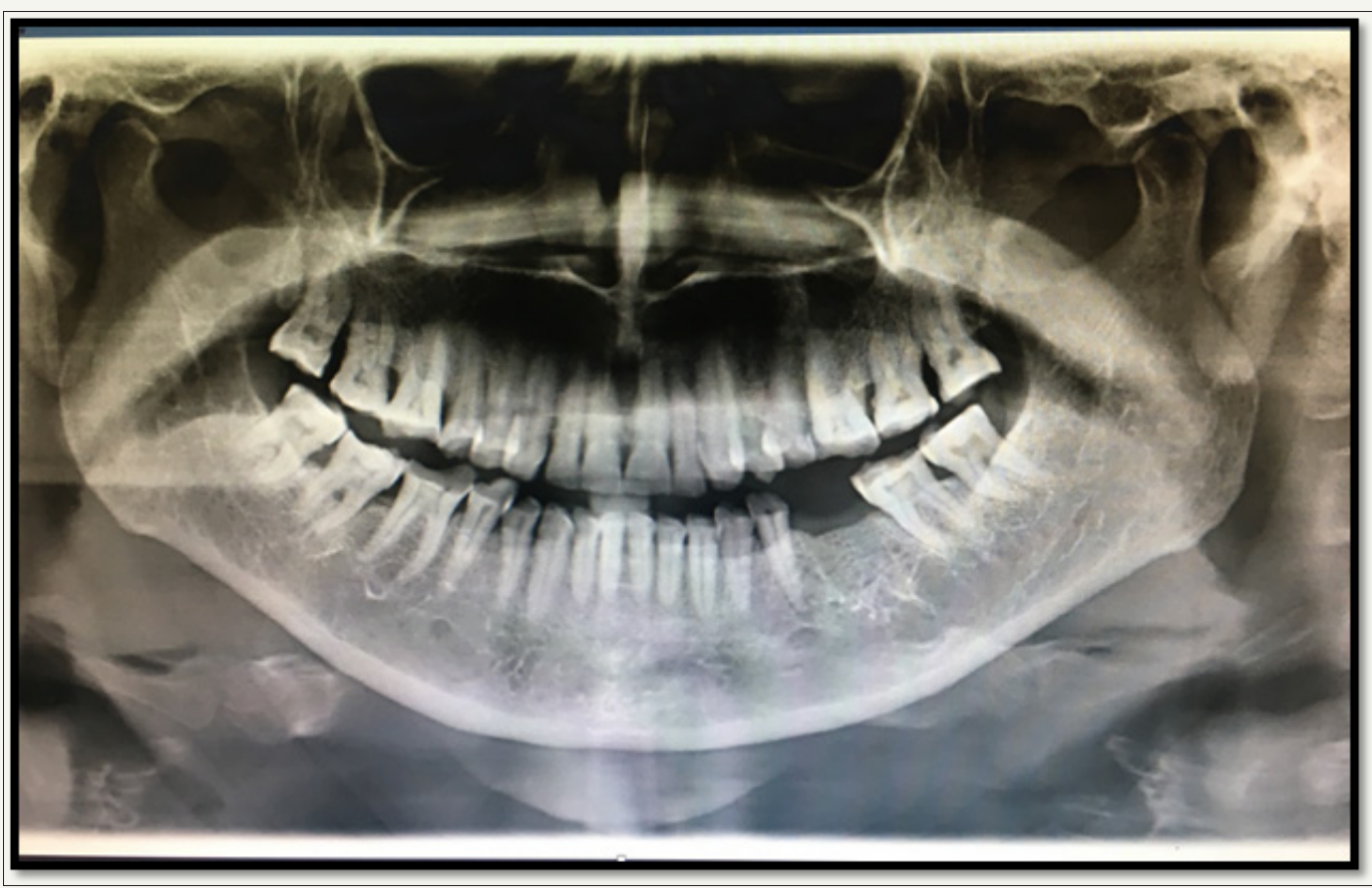

Figure 4: Orthopantamogram with apparently normal condyles and TMJ with generalize.

\section{Discussion}

Dystonia is the manifestation of involuntary lasting severe muscle contractions, which lead to rhythmic and atypical movements in different parts of the body. Based on the affected area, dystonia can be anatomically categorized as focal (affecting one or two parts of the body), segmental, multifocal, and generalized ${ }^{1}$. It can also be categorized based on etiology as primary dystonia which is idiopathic or inherited; while, secondary dystonia advances after traumatic or surgical incidents, brain diseases, and medications [2]. Head and neck dystonia manifest clinically by the presence of involuntary sustained, forceful muscle contractions, and characteristic rhythmic movements and abnormal posture [3]. Craniocervical manifestations of dystonia affect the person's quality of life by interfering with the ability to speak and swallow and in social interaction. Primary dystonia has a prevalence of 3.4 per 100,000 as generalized dystonia [4]. Oro Mandibular Dystonia is considered as a focal dystonia involving mouth, jaw, and tongue, manifested by involuntary muscle contractions producing repetitive, patterned movements of the involved structures $[1,5,6]$. OMD is classified and categorized with presenting clinical sings of repititive jaw opening, jaw closing, jaw deviating, or lingual dystonia, or a combination of these. Combination of OMD, belpharospasm (abnormal eye twitching), and dystonic movements of the upper face is called Meige's syndrome [1,7-9].

Other terms for OMD are orofaciomandibular dystonia, orofacial buccal dystonia, lingual dystonia, jaw dystonia, cranial dystonia, and adult onset facial dystonia $[1,4,5]$. The prevalence of OMD has been reported to be as high as $6.9 / 100,000$ cases and the incidence has been reported up to 3.3 cases per million [6]. The mechanism and the cause of OMD are not well known. OMD may occur per se as a neurological disorder (with or without a hereditary history), or as secondary to certain drugs (anti psychotics) or disorders such as trauma or Wilson's disease $[4,9]$. Central nervous system (CNS) trauma, neuroleptic exposure, hypoxic brain damage, metabolic disorders, and ischemic or demyelinating lesions in the upper brain stem can be related to OMD [7]. It has been suspected that dental and oral surgery interventions or treatments can be associated with the onset of dystonia [10-16]. In the present case, the patient was apparently asymptomatic concerned to the repetitive jaw movements yet noticed the change in occlusion following the dental extraction (a month before). However, considering the chronicity in the possible etiological factors for this condition, dental induced etiology was ruled out. The diagnosis of OMD is clinical and complicated, since it presents in various forms and severities. It responds to no diagnostic medical test; hence, the diagnosis is based on history, neurological examination and confirmation by intramuscular electromyography (EMG) [1]. The differential diagnosis includes TMJ disorders (like bruxism or spontaneous condylar dislocation), hemifacial spasm, and psychological disorders [1]. The symptoms of OMD can get worse by emotional factors, which is among the reasons for the delayed diagnosis. In the aforementioned case report, the patient denied the clinical finding to be considered and hence further investigations. From the evaluation of the patient, review of systems and presenting signs which were not attributed to any other lesional, traumatic or organic cause, a clinical diagnosis of oromandibualar dystonia was made.

Treatment of OMD is multi-disciplinary and varies from one patient to another. There is inadequate evidence-based information about the efficacy of various medications currently being used for dystonia [7]. Nevertheless anticholinergic, baclofen, benzodiazepines, antiparkinson drugs, anti-convulsants, 
carbamazepine, dopamine receptor antagonists, levodopa and lithium are some of the agents used to manage OMD [12]. Physiotherapy is thought to promote brain rewiring over time, so as to reduce the dystonic movements [17]. Botulinum neurotoxins (BoNT) injection, a promising therapy for treating OMD, is a potent neurotoxin that blocks the release of acetylcholine at the presynaptic junction, resulting in temporary chemical denervation of skeletal muscles. BoNT injections can leave even better effects if guided with EMG, which allows longer intervals between the BoNT injections. BoNT injection is mainly technique dependent. Depending on the dose of therapy, jaw weakness or tremor, loss of smile, and dysphagia are the expected side effects. BoNT may be immunogenic, and some patients may develop secondary non- responsiveness following multiple injections. Swallowing difficulties, speech problems, and excessive muscle weakness have been reported in the treatment of lingual dystonia by BoNT [15-19]. Yoshida et al. [20] found Muscle Afferent Block to be highly effective on OMD; although, it had no effect on patients with dyskinesia symptoms. Thus, OMD and oral/orofacial dyskinesia might differ in physiopathology [20]. There is no specific surgical therapy for OMD, and the role of CNS procedures has not been proven. In some patients with peripherally induced OMD arthroscopy and other TMJ surgeries were done with no useful outcome; the conditions got even worse in some cases $[12,15,21]$. Psychosocial and occupational therapy, support groups participation, cognitive behavioral therapy, and deep brain stimulation surgery were also designed to reduce the hyperactivity of the muscles [12]. Treatment modalities pertaining to dental rehabilitation including acrylic mimics of sensory tricks, implant supported dentures and removable appliances were also suggested with a varying range of treatment outcome in the literature [22-24].

\section{Conclusion}

The Ultimate burden on oral health is of significant interest to the dentist as a vast range of dental implications are reported in the past literature in the form of attritions, TMJ dysfunctions, increased caries risk, denture instability, loss of multiple teeth, alveolar atrophy, damage to restorations, and marginal to advanced periodontitis. It is important for the dental surgeons to be familiar with oromandibular dystonia, as it can develop after dental treatment. Very few reported cases in Indian population exist as often these disorders are labeled psychogenic or characterized as temporomandibular disorders. Henceforth the speciality dental surgeons should identify such often misdiagnosed cases of suffering patients often pushed to the realm of mental illness. Although prompt diagnosis in the presented case could explain the details of the persisting condition in the patient, denial of the facts and findings hindered the further investigations and appropriate management. Thus, a thorough knowledge about the neuromuscular related pathophysiologies is a mandatory for the clinicians of any speciality to generate awareness and enlighten the patients towards appropriate management of existing diseased conditions.

\section{References}

1. Raoofi S, Khorshidi H, Najafi M (2017) Etiology, diagnosis and management of oromandibular dystonia: an update for stomatologists. Journal of Dentistry 18(2): 73-81.

2. Watt E, Sangani I, Crawford F, Gillgrass T (2013) The role of a dentist in managing patients with dystonia. Dent Update 40(10): 846-848.

3. Gn S, Nag A (2017) Management of oromandibular dystonia: A case report and literature update. Case reports in dentistry doi: $10.1155 / 2017 / 3514393$.

4. Lee KH (2007) Oromandibular dystonia. Oral Surg Oral Med Oral Pathol Oral Radiol Endod 104: 491-496.

5. Khan J, Anwer HM, Eliav E, Heir G (2015) Oromandibular dystonia: differential diagnosis and management. J Am Dent Assoc 146(9): 690693.

6. Bakke M, Larsen BM, Dalager T, Møller E (2013) Oromandibular dystonia-functional and clinical characteristics: a report on 21 cases. Oral Surg Oral Med Oral Pathol Oral Radiol 115(1): e21-e26.

7. Jinnah HA, Teller JK, Galpern WR (2015) Recent developments in dystonia. Curr Opin Neurol 28(4): 400-405.

8. Gupta S, Bhagwati BT (2010) Oromandibular dystonia- meige's syndrome: Report of a rare case with review. J Indian Acad Oral Med Radiol 22(4): 218-220.

9. Esper CD, Freeman A, Factor SA (2010) Lingual protrusion dystonia: frequency, etiology and botulinum toxin therapy. Parkinsonism Relat Disord 16(7): 438-441.

10. Chidiac JJ (2010) Oromandibular dystonia treatment following a loss of vertical dimension. Dent Update 38(2): 120-122.

11. Jang SM, Cho YC, Sung IY, Kim SY, Son JH (2012) Oromandibular dystonia after dental treatments: a report of two cases. Korean Assoc Oral Maxillofac Surg 38(6): 379 -383.

12. Thorburn DN, Lee KH (2009) Oromandibular dystonia following dental treatment: case reports and discussion. N Z Dent J 105(1): 18-21.

13. Sutcher HD, Underwood RB, Beatty RA, Sugar O (1971) Orofacial dyskinesia. A dental dimension. JAMA 216(9): 1459-1463.

14. Thompson PD, Obeso JA, Delgado G, Gallego J, Marsden CD (1986) Focal dystonia of the jaw and the differential diagnosis of unilateral jaw and masticatory spasm. J Neurol Neurosurg Psychiatry 49(6): 651-656.

15. Peñarrocha M, Sanchis JM, Rambla J, Sánchez MA (2001) Oral rehabilitation with osseointegratd implants in a patient with oromandibular dystonia with blepharospasm (Brueghel's syndrome): a patient history. Int J Oral Maxillofac Implants 16: 115-117.

16. Balasubramaniam R, Rasmussen J, Carlson LW, Van Sickels JE, Okeson JP (2008) Oromandibular dystonia revisit-ed: a review and a unique case. J Oral Maxillofac Surg 66(2): 379-386.

17. Jinnah HA, Factor SA (2015) Diagnosis and treatment of dystonia. Neurol Clin 33(1): 77-100.

18. Maestre-Ferrín L, Burguera JA, Peñarrocha-Diago M, Peñarrocha-Diago M (2010) Oromandibular dystonia: a dental approach. Med Oral Patol Oral Cir Bucal15(1): e25-e27.

19. Schneider R, Hoffman HT (2011) Oromandibular dystonia: a clinical report. J Prosthet Dent 106(6): 355-358.

20. Yoshida K, Kaji R, Kubori T, Kohara N, Iizuka T, et al. (1998) Muscle afferent block for the treatment of oromandibular dystonia. Mov Disord 13(4): 699-705.

21. Yoshida K (2006) Coronoidotomy as treatment for trismus due to jawclosing oromandibular dystonia. Mov Disord 21(7): 1028-1031. 
22. Blanchet PJ, Rompré PH, Lavigne GJ, Lamarche C (2005) Oral dyskinesia: a clinical overview. Int J Prosthodont 18(1): 10-19.

23. Yoshida K (2017) Surgical intervention for oromandibular dystoniarelated limited mouth opening: Long term follow-up. J Craniomaxillofac Surg 45(1): 56-62.
24. Sibley DM (2013) Restoring the edentulous patient with oromandibular dystonia: Treatment planning considerations and a review of the current literature. 71(9): 51.
For possible submissions Click Here

Submit Article

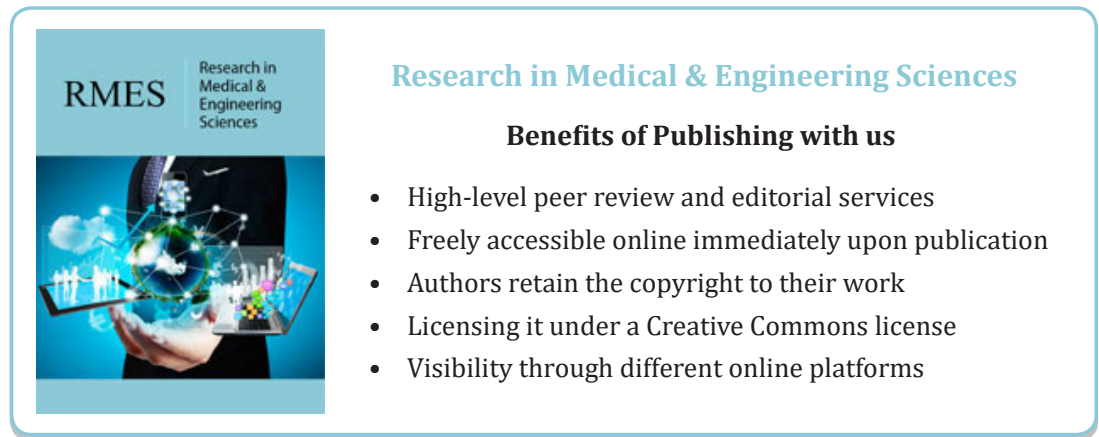

